\title{
KILLING MOSQUITOES
}

\section{Think before you swat}

\author{
Marcus Hall and Dan Tamir
}

Am I not mosquito enough to out-mosquito you?

-D.H. Lawrence, “The Mosquito” (1920)

Global warming is ushering us into a new mosquito epoch. Ready or not, mosquitoes are coming faster than before, both indigenous and non-, human-biting and not, disease-carrying and sometimes-disease-carrying. What are we to do with these buzzing creatures, and what has been done with them so far? Usually perceived as a pest or at least as a nuisance, their mere presence often prompts us to take action. Are we able to control, or locally exterminate them, and with what side effects? Or is it more realistic to admit that the three most threatening mosquito genera-the disease carrying Aedes, Anopheles and Culex-are really controlling us? In recent years, yellow fever has kept spreading even as malaria has been retreating, but over half of the world's population is still exposed to these and other dangerous mosquito-carried diseases which also include dengue, West Nile, chikungunya and Zika. Control them we should; we must do, if we are to avoid the next pandemic and survive our mosquito-borne Anthropocene. COVID-19 has been humanity's latest collective horror, but across deep time, and likely into the foreseeable future, mosquitoes will be responsible for inflicting incalculably greater degrees of suffering and anguish.

But there are important reasons to protect mosquitoes, and not just because these creatures are amazing products of millions of years of evolution-since protecting them may in some instances assist us in the battle against various human diseases. Most obviously, we may want to save some mosquitoes for the simple reason that one needs to preserve a few of them in order to figure out how to kill the rest of them-with other practical reasons detailed below. Yet more subtle justifications for saving mosquitoes centre, for instance, on food web dynamics, whereby in our efforts to poison these creatures, or disrupt their 
habitat, or rearrange their DNA, we may, through ecological loops, actually cause damage to other biological entities, such as mosquito predators, and end up increasing a mosquito's fitness and its ability to multiply and spread across the earth. Perhaps the sciences of mosquito control, or certain sectors of them, have not yet advanced to a stage that we can trust.

Some years ago, Nature journalist Janet Fang posed the simple but powerful question about what the ecological consequences might be of eradicating mosquitoes (Fang 2010). A concerted campaign across the twentieth and twentyfirst centuries, after all, has been dedicated to this very goal. In sifting through the evidence, Fang's final answer is that in the case of this blood-sucking insect, humanity and even ecosystems could probably get along just fine without it. She reports on the views of one ecologist who feels that mosquitoes could readily be replaced in the food web, with many mosquito predators eventually able to switch to moths or houseflies or other sources of food. Although she outlines a host of possible disruptions stemming from the disappearance of mosquitoes, such as the loss of their pollination activities and other ecosystem services, she concludes by quoting entomologist Joe Conlon who believes that ecosystems "will hiccup and then get on with life. Something better or worse would take over." Or as Conlon elaborates in his own blog, "I would rather eat raw onions and celery for the rest of my life if I could do away with the little bastards" (Conlon 2011).

In these introductory pages we highlight some of the main arguments for saving mosquitoes, before reminding ourselves of vital reasons for setting out to control and eradicate them. Ours is not a comprehensive list, and our main goal here is to stimulate readers to begin thinking about the many reasons for saving or else exterminating these creatures, while outlining some pressing points that will be taken up in subsequent chapters. Confronting this question of how far we can, or should, pursue the goal of mosquito elimination is our central purpose of this book, seeing if there may be a kind of peaceful coexistence that we can achieve with these creatures. In the end, rather than pushing an ultimatum that it must be us or them, can humanity promote and practice a kind of "Mosquitopia" with these little humming creatures, humanity's most dangerous companions? Could we develop a relationship with this insect that will allow healthy cohabitation?

The project of searching for and identifying a possible harmonious coexistence involving humans and mosquitoes has implications for the lives and lifestyles of many millions of people affected by mosquito-borne pathogens. But it also becomes a crucial test case for identifying the proper place of people in the natural world. Although mosquitoes are amongst the most intimate of animals accompanying humanity across millennia, similar questions may be asked about scores of other species, including such charismatic ones as bears, dolphins, rhinos and orangutans, whose prospects are shaky, to say the least. The majority of human-animal interactions, as well as the greater part of the sixth species extinction crisis we are experiencing, involve many smaller jewels in the treasure box 
of creation. The mosquito then becomes one example, and an emblematic one to start with.

\section{Some reasons for saving mosquitoes}

The first reason for making our truce with mosquitoes is strategic. We must remind ourselves that we are ultimately battling diseases, not mosquitoes, and that there may be more effective, more economical, and more ethical ways to do this than killing that little, ubiquitous insect. Malaria, for instance, once emanated from swamps and the bad air they produced, although with more evidence it became clear that mosquitoes, rather than effluvia, were the vectors (or transmitters) of the malaria parasite. Should we be putting greater efforts into battling this microscopic Plasmodium rather than the carriers of them, as by developing more effective malaria drugs? Or should we be focusing at stillsmaller levels, as by managing the chemical reactions set in motion by the Plasmodium, or else by treating the resulting symptoms, to let the body take care of itself? Two generations ago, zoologist Marston Bates considered the use of the powerful insecticide, DDT, to be the "sledge hammer approach to mosquito control" since this chemical caused so much collateral damage to other living things, from birds and fish to desirable insects such as bees (Bates 1953). An early anti-malarial medication such as Atabrine was itself a sledge hammer approach in the human bloodstream, since people often felt quite nauseous after taking it. With the ecological knowledge accumulated and the microbiological techniques developed since then, isn't it more realistic to see all population-level control techniques, whether applied to wetlands or to human bodies, as sledge hammers? And as our understanding of mosquito-borne diseases becomes more precise and accurate, the surgical response of today may seem like a sledge hammer tomorrow.

Eradicating any of the mosquito-borne diseases may therefore necessitate the extermination not of mosquitoes, but of the pathogens themselves. The malariologists' phrase of "anophelism without malaria" (or, the presence of anopheles mosquitoes without malaria) is known in many countries where the disease practically disappeared decades ago. Notwithstanding the differences in ecologies of other vectors and their transmission mechanisms, can we aim at parallel situations of "aedeism without dengue" or "culexism without West Nile"? The distancing of a pathogen from a human population - and even its total elimination-may be less challenging and less problematic than insect eradication. Because there are pros and cons to every health remedy, we need to return to ecological principles as well as cost-benefit analyses, before marching forward with any one strategy for disease control.

A second justification for preserving mosquitoes centres on medical reasoning. Modern epidemiological research reveals that there may be important benefits to maintaining discrete, residual levels of pathogens in a population so as to maintain immunological signals that our bodies can react to and maintain resistance 
against. Madagascar provides a telling example: when malaria was largely eradicated from parts of that large island between 1960 and 1980, it returned there several years later with more deadly virulence. Maintaining some mosquitoes there, and with them the diseases they transmit, means that human physiologies would not become naively adapted to an environment only temporarily free of this or that disease (Carter and Mendis 2002). A related issue is that certain kinds of less dangerous malaria can provide a degree of protection from more dangerous forms of it: a person infected by Plasmodium vivax is often given some resistance against being infected by the more lethal Plasmodium falciparum (Snounou and White 2004). In this case, a normal, mosquito-transmitted vivax malaria can be the lesser evil of contracting falciparum malaria.

There are also important ecological reasons for keeping mosquitoes buzzing, based on arguments pointing to the special role of these arthropods in ecosystems. Metric tons of flying biomass certainly alter natural processes, whether as foodstuff for other organisms or as modifiers of animal behaviour, as in the case of caribou and Homo sapiens who move or migrate to avoid them. Enormous numbers of friendly insects fall victim to the many projects of mosquito control (Török et al. 2020). Mosquitoes carry parasites and pathogens, not only to humans, but also to many other mammals, birds and reptiles. Microbes transmitted by mosquitoes to bats help control the numbers of the latter, thereby controlling the human diseases spread by these winged mammals. Moreover, it is only female mosquitoes that transmit pathogens by feeding on blood while their male counterparts generally subsist innocuously on plant nectars. Some mosquitoes even control other species of mosquitoes, since certain adult species feed on larvae of other species (Roux and Robert 2019). These mosquito-borne benefits are a sampling of reasons for maintaining at least some mosquitoes in ecosystems, or else bringing many of them back if drastically reduced.

Yet another rationale for saving mosquitoes is the evolutionary one. As an example, parasites and hosts coevolve, sometimes with beneficial results for both, since both members in such relationships generally become more tolerant of the other through time. Or at least this is the argument of Nobel laureate Joshua Lederberg for why the virulence of parasites can diminish over decades and centuries (Méthot 2012). Cautious hands-off approaches to vector control therefore allow nature to take its course, with harmful results balanced increasingly by beneficial ones over the longer run. There appear to be indispensable long-term roles for many of our bodily symbionts, and human acts of interference in their transmission may, over the short or long term, cause more harm than good.

From a more cognitive perspective, we may identify ethical reasons for leaving mosquitoes alone. Do humans have the right to kill or exterminate other creatures - or the right to transform or disrupt whole ecosystems? Is it justifiable to exterminate when we are still quite unsure how the many parts of an ecosystem fit together? If we are placing humans at the top of the pyramid of creation, what does that tell us about ourselves and our place in the future? To date, we 
have never been able to rid islands or continents of mosquitoes despite dogged efforts to do so-or at least not for very long: what makes us believe we can exterminate them now? More often than not, hubris has been the rule, not the exception, in describing humanity's attitude towards managing the non-human world. The question of eliminating mosquitoes should not be undertaken while being detached from wider global and environmental contexts. Our current and ongoing sixth mass extinction episode is gathering force, already eliminating thousands of species, while impoverishing the biosphere and annihilating millions of years of evolution. Such dramatic changes carry with them exceptional uncertainty, notably as potential adverse repercussions, be they ecological, political and/or economic. A key general guideline is the precautionary principle which may be phrased: "in case of doubt, stop." Such precautions may be more applicable to biodiversity preservation than to almost any other environmental problem (Myers 1993).

The scope of the extinction crisis may contribute to a notion of helplessness, by assuming that the saving of a few species of insects is a marginal concern that would not change much. Yet the severity of the crisis may pose a super-premium on applying the precautionary principle even more broadly: the burden of proof is on those aiming at eradication-call it deliberate extinction. As in so many other issues, a decent portion of modesty is a key strategy for making the right decision.

And yet - is it even thinkable that humans can propose a moral justification for not seeking every means possible to curtail or eliminate disease-spreading organisms? Is it ethically responsible to pay so much attention to insects and their needs when humans are made sick by them, and even die? Can it be advisable, or right, to rely on expert opinion, when the individuals directly affected by antimosquito treatments hold different viewpoints? Involving local communities in decisions about the tactics and timing of mosquito control is a relatively new recommendation of pest-control agencies.

From an economic perspective, millions of Euros and thousands of researchers are now dedicated to finding more effective vector control. In terms of spending efficiency, should these limited resources be diverted to other measures, such as bed nets, tighter houses, better equipped hospitals, and health education? Mosquito control is one of many health measures, and may merit lesser priority depending on circumstance or period. Yet another economic issue focuses on the potential utility of mosquitoes to industry or science; for example, mosquitoes can detect minuscule quantities of carbon dioxide, and produce amazing anti-coagulants, with both traits suggesting entrepreneurial opportunities unless these are curtailed by exterminators.

Last but not least is the aesthetic dimension of mosquitoes. Insects in general, and mosquitoes in particular, are exquisitely engineered organisms, supremely adapted to their various roles, and elegantly effective in carrying them out. We cannot help but admire them, even paint them, sculpt them, and remark on their carefully tuned soundscapes. Mosquitoes manage to pair with each 
other by matching the frequencies of their beating wings, so that sound artists can amplify and project these harmonic vibrations to demonstrate this insect's underappreciated acoustic attributes (Borrell 2009, BBC News 2013). Even with all the harm these insects cause, they are also beautiful, inspiring creatures. Of course, beauty by itself cannot be a justification for sparing harbingers of disease; but it should certainly prompt us, at the very least, to ponder saving rather than squishing these little humming beasts.

\section{Key reasons for killing mosquitoes}

Of course, beyond the many motives and justifications for protecting mosquitoes, there are also urgent reasons for ridding ourselves and the planet of these creatures. The list here is shorter, but may be just as powerful. The first and obvious reason is human health. Despite reasons for saving mosquitoes some of the time, or at least saving certain mosquitoes under certain situations, there remains a dire need to eradicate the disease-carrying varieties, utilizing even extreme measures to accomplish this goal — since the mosquito may be humanity's deadliest foe (Spielman and D'Antonio 2001, Winegard 2019). A crucial reason why some mosquito-borne diseases are not more pervasive today than in past decades, at least in some regions, is that former mosquito controllers were reasonably successful in their missions, bringing mosquito numbers down long enough so that the pathogenic virus, bacteria and protozoa they carried dropped below dangerous threshold levels. Although the percentages of people contracting malaria around the world are lower today than ever before, outbreaks of dengue and yellow fever are more serious in many areas than they were a decade or two ago (Mosquito Reviews 2020, WHO 2014, Jones 2012). We must assume that pandemics of mosquito-borne diseases could be brought into better control by intensifying the many anti-mosquito campaigns being waged around the world.

A second and related reason for stepping up mosquito control centres on practical motives: the project of exterminating mosquitoes allows us to avoid other, undesirable health or economic side-effects of dealing with these diseases, such as ingesting nauseating medications or diverting resources away from other pressing social issues. A case in point is when malaria-exposed soldiers and civilians during World War II avoided taking their prophylactic quinine or Atabrine because of the sickening side effects of this drug (Hall 2010). Finding a magic bullet that removes mosquitoes from ecosystems may therefore have ulterior beneficial consequences beyond curtailing disease, including the ability to redirect healthcare to combat other illnesses. Systematic sterilization of mosquitoes might allow wetlands to remain wet, for example, since draining them would no longer be required to disrupt mosquito habitat.

The control of mosquitoes might also be justified through a consideration of striving for better ecosystem management. Our human-altered biosphere means that mosquito numbers and their distribution are no longer natural, no longer in balance, so that human action is required to bring those balances into better harmony. 
After all, today's abundance of mosquitoes and their accompanying pathogens can be traced in part to human agency. Since irrigation projects, say, or water-filled containers and discarded car tyres have favoured mosquito breeding in many parts of the world, then we can be better justified in seeking ways to diminish mosquito numbers. Here, Stewart Brand's dictum that "We are as Gods, and may as well get used to it" holds true for mosquito management (Brand 1968).

In a related issue, it seems appropriate in the context of human-mosquito relations to mention the Anthropocene: the current geological era in which Homo sapiens is modifying the entire planet. No place on earth has been spared by our alteration of ecosystems and our movement of creatures, with mosquitoes being part of that trend. The long coexistence of humans and mosquitoes made it only natural that we bring our winged companions with us across oceans and continents, while setting the table for them and preparing comfortable breeding sites (Kennedy and Lucks 1999, Boomgard and 't Hart 2010). After Europeans settled in certain areas of coastal South Africa, for instance, mosquito swarms arose where they were once rare: one explanation is that newly erected metal roofs on houses concentrated rainfall into puddles, thereby multiplying mosquito habitat and so mosquito-borne disease. A rational human response would therefore aim at resetting environmental equilibria, seeking to shrink mosquito habitat there. Such an argument could be used for justifying efforts to exterminate invasive alien Asian Tiger mosquitoes (Aedes albopictus) that never used to buzz across the Americas and across Europe before being introduced there by humans, but are now propagating at least 20 threatening diseases in these continents (Bhaumik 2013). Altering stream ecology by introducing alien Gambusia fish for slurping up mosquito larvae, as has already been carried out in many areas of the world beyond their native North America, may be part of the necessary quest to reengineer the earth.

In a different light, there are also strong justifications for killing mosquitoes from the perspective of economic development. Poverty levels tend to worsen in a malarial environment, for instance, but evidence is inconclusive about whether mosquito-borne diseases are causes or effects of this impoverishment. While some experts claim that malaria blocks a country's economic development to result in poverty (Gallup and Sachs 2001), others assert that it is the continuous and deep impoverishment in communities that fosters the spread of malaria (Packard 2007). The effects of disease on development may hinder globalization, polarize private and public sectors, and disrupt international trade. The project of killing mosquitoes can be a catalyst for escorting a nation out of dependency.

A last-but-not-least reason for exterminating mosquitoes is human comfort. Pesky and nuisance mosquitoes drive people inside or away from their favourite places. In areas where mosquito-borne diseases are not a threat, the act of removing these bloodthirsty insects would still seem a good reason to continue funding mosquito-control agencies. After all, clearing mosquito swarms allows other organisms easier access to their grounds, including humans. Coastal wetlands, as in New Jersey, were virtually uninhabitable until early-twentieth-century 
drainage measures decimated mosquito populations and brought up land values (Patterson 2009). There is also evidence predicting that mosquitoes that currently do not feed on people may, with climate change or other stressors, develop a preference for human blood to then transmit the pathogens they carry (Rose et al. 2020). So, yes, even when the level of nuisance is "bearable," the physical and mental well-being of humans cannot be ignored, especially if we want to mobilize fellow men and women to confront other environmental and health challenges.

The case for killing mosquitoes here, or conserving them there, therefore goes to the core of what it means to be human in the natural world. The above points offer just a sampling of reasons for supporting either side of the question about what should be done with these biting insects-and certainly there are many other reasons to be added. The following chapters take up these reasons, expanding them while suggesting still other areas that need to be addressed. How we interact with, show mercy for, declare war on, or learn to live with our most dangerous game becomes a parable of our future on this planet. "I do favor insect control in appropriate situations," the great naturalist Rachel Carson once declared, even if the question that obsessed her to the end of her days was "whether any civilization can wage relentless war on life without destroying itself, and without losing the right to be called civilized" (Carson 1962a, 1962b). Mosquitoes present us with a supreme case for identifying ways to simultaneously promote people and pests on the same earth. A "Mosquitopia" is therefore a balanced relationship between ourselves and our main insect adversary that can permit us to both survive and prosper into the next epoch. Acknowledging the benefits and rights of our pathogens, parasites and predators (large and small), and then adjusting our lives and lifestyles to make room for them, is the pathway to a better existence.

\section{What is in this volume}

The book you are holding includes five parts for exploring the general question of whether we could, or should, eliminate mosquitoes. A vast experience demonstrates that insect controllers have been able to eliminate some mosquitoes, in some places, some of the time, but they have never been able to eliminate them completely to finally achieve species eradication. To date, the eradication of a pest has been achieved for only two microbial creatures, these being the viruses that cause smallpox and rinderpest - and only a few laboratory samples of such viruses survive to this day. Yet in the case of mosquitoes, we need to ask whether we could ever manage to eradicate this creature, and just as importantly, whether we would want to see this creature disappear forever. Even if eradicators and controllers focus their efforts on just a handful of the deadliest of some 3,500 mosquito species, as several authors maintain in the following chapters, we still need to ask hard questions: are the resources dedicated to their elimination better diverted to other health projects; are the inevitable collateral damages of the 
battle worth the cost; and are we ever able to know what is best for the earth's ecosystems? Even the project of pest control, as the first step toward pest eradication, requires us to think hard about resource allocation, collateral harm and the limits of our human abilities.

The book's first part prepares the reader for subsequent chapters by including essential information about the mosquito, its habits and traits-especially those related to its often painful encounters with humans. Here the reader can ponder examples of the intricate and elaborate ways in which humans and mosquitoes interact and the manners in which their life paths are intertwined. Even entomologists and anthropologists will find new ideas here that will help them reflect on the rest of the book. The second part is historical in character, drawing attention to past case studies and experiences. These mosquito stories stretch from Africa to England, from North America to Malaysia, spanning zones tropical to temperate; from distant eons of prehistory to the twenty-first century. This is not an attempt at compiling a comprehensive social or environmental history of mosquitoes; rather, this retrospective part presents examples of evidence and experience for elucidating issues worth considering when thinking about and dealing with mosquitoes today.

The book's third part centres on what can be termed (not without some provocation) "the enemy" and our perception of it as such. Beyond the obvious view of the mosquito as humanity's clear and present danger, this part challenges that view. How deeply are we committed to mosquito-as-adversary, and what may be done to alter that perspective? This buzzing insect feeding on blood may be anthropophilic (human-loving), but it may never become domestic (humanobedient). The next, fourth part is focused on us, humans: how do we react to mosquitoes? How should we understand them and put them in context within our larger living planet? Mosquitoes present us with nuisance and pathogens, and one wonders to what extent we can expect to live out our lives without confronting hardship and disease. The fifth and concluding part presents recent techniques and practices used for coping with mosquitoes and mosquitoborne diseases, some of which involve genetic engineering (such as CRISPRCas9 technology) along with applied evolution. By reviewing conduct and experimentation in the laboratory and the field, these chapters show what is now being achieved, while putting the results in wider ecological and social contexts. Building successful new relationships with mosquitoes demands technological and scientific understanding to be carried out with social and political sensitivity.

\section{The world is warming-and buzzing}

Mosquitopia, as a project, emerged from INFRAVEC2 (2017-2020), a European Union Horizon 2020 research initiative aimed at understanding and controlling disease vectors, especially mosquitoes. Weighing mostly on the natural sciences, especially for identifying ways to repel or exterminate these creatures, a small part of INFR AVEC2 was also dedicated to examining the human side of dealing 
with mosquitoes. Our mission as humanists and social scientists was to reflect on these creatures and suggest ways to better understand interactions between species, human and insect.

A first consideration of our collaborative project suggests that its mere existence is already a success, by providing more evidence of the necessity and feasibility of bridging the gap opened between the natural sciences on the one side, and the social sciences and humanities on the other. The separation of these "two cultures" has been one of the gaps of thinking throughout the twentieth century, precisely at a time when humans were conducting concerted campaigns against the mosquito (Snow 1959). Despite brilliant scientific innovations combined with enormous technological progress, such campaigns have hitherto not been crowned total successes. We may plausibly assume that strengthening the connections between natural and humanistic sides of our encounters with the mosquito will contribute to achieving more satisfactory results. Our multi-disciplinary collaboration might be considered an "environmental humanities of the mosquito," since the issues raised require environmental insights from many disparate fields (Hall et al. 2015). Mosquitoes evoke health questions, but also ecological, ethical, anthropological, historical and literary questions, with the theme of our book being that crucial answers to the mosquito question require the full range of human inquiry.

Here, we acknowledge with gratitude the financial and organizational contributions from INFRAVEC2, EU Horizon 2020 for helping develop this project, along with crucial support received for our initial symposium from the Rachel Carson Center for Environment and Society (Munich), as well as in our later publication stages from the University of Zurich, Department of Evolutionary Biology and Environmental Studies and the Swiss National Science Foundation. We also thank the many institutions that have supported our authors in developing their chapters, and Douglas Da Silva for developing the index and Jayanthi Chander for project management.

We close this brief introduction by reflecting on Italy's famous Sardinian Project (1946-1951), which in the annals of mosquito control stands out as perhaps the premier instance in which men, women and their machines, together with metric tons of insecticide, composed mostly of DDT, were unleashed in a concerted action against Anopheles labranchiae, this island's most notorious malaria mosquito vector. At the height of this five-year campaign, some 30,000 mosquito eradicators fanned out across the Mediterranean island, draining wetland habitat, straightening meandering rivers, introducing Gambusia mosquito fish, and spraying DDT-by hand and airplane, in liquid and powder form - in every open well, on every watery spring, inside almost every house and stall. The goal of the Sardinian Project was to eliminate across this large and rugged island every single An. labranchiae, whether in larva or adult form, so that this mosquito would never again fly here, and its future control would never again be necessary, finally extinguishing the age-old scourge of malaria from its shores. The campaign's final report explained that

Eradication was regarded as an objective which could be attained only through a complete concentration of effort. The end result was to be either 
success or failure, eradication or failure to eradicate, and any additional objectives would weaken the principal one.

(Logan 1953)

But in the end, following five seasons of dust and drudgery, spraying and respraying, and then scouting for surviving mosquitoes to spray again, it became clear that An. labranchiae had not been completely eliminated, and that these insects would continue to buzz into the foreseeable future. Meticulous field work demonstrated that Sardinia's eradicators had come very close to achieving their goal, calculating that $99.936 \%$ of this species of mosquito had been exterminated from the island. Yet by the standards of the Rockefeller Foundation that oversaw the campaign, the Sardinian Project was deemed a "failure" because eradication had not been achieved. Malaria had been expunged from the island, but a few An. labranchiae remained, with the survivors surely showing DDT resistance and passing this resistance to their offspring that would continue propagating across the land. Here there was failure to eradicate the mosquito, but success at controlling the main disease it carried (Brown 1998).

In our own more humble mosquito project that follows, we hope that any success of bringing together humanists, social scientists and natural scientists

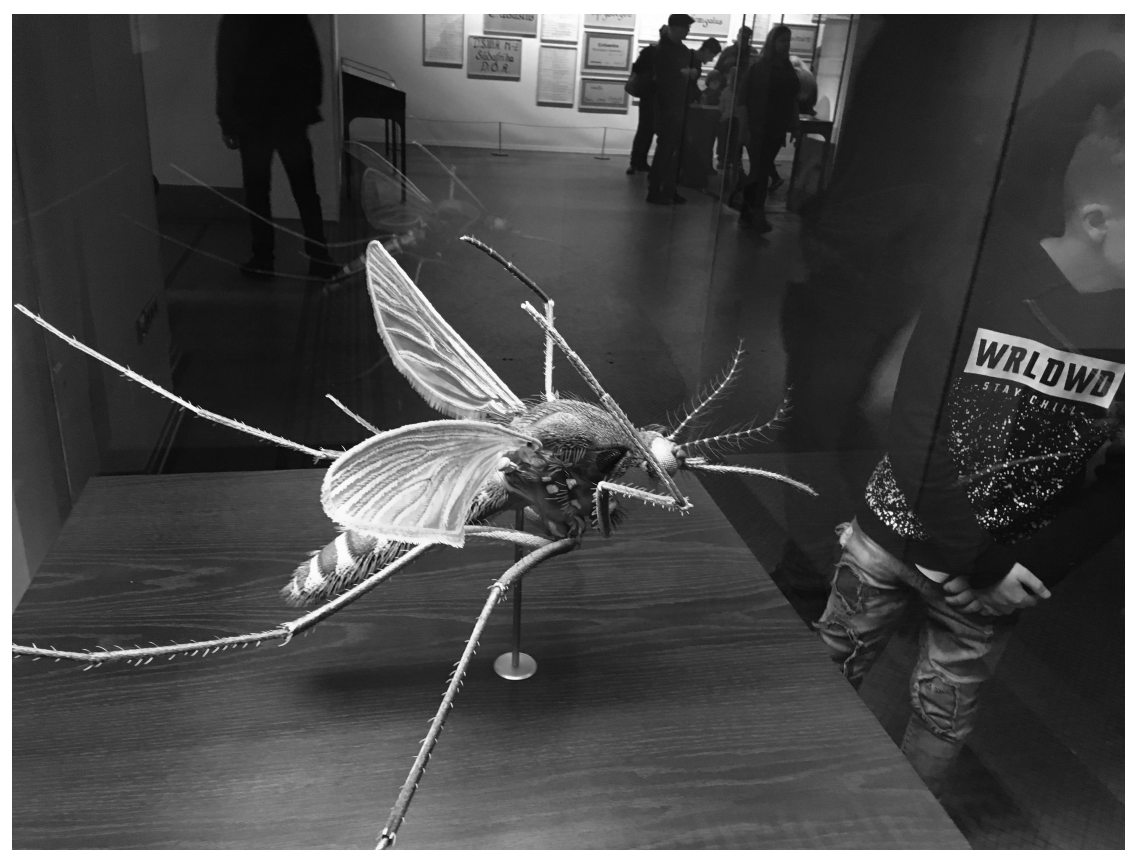

FIGURE 1.1 A $60 \times$ life-size model of the common house mosquito (Culex pipiens), by Alfred Keller 1937. Museum für Naturkunde Berlin. Photograph by Marcus Hall. 
will not be judged as narrowly as was the Sardinian Project. We believe that environmental humanities of the mosquito can show pathways to success, or at the very least, pathways that need to be taken to avoid failure in understanding the mosquito. The project of controlling and even eradicating mosquitoes must be scrutinized from many perspectives in order to judge whether it is technically feasible, ecologically compatible and ethically reasonable (Angelone et al. 2020). In the end, judging the success or failure of Mosquitopia will ultimately lie with the reader, and how the reader may build new relationships with the mosquito.

\section{Bibliography}

Angelone, Samer, Marcus Hall, and Dan Tamir. 2020. "Mosquitopia? Peaceful coexistence between humans and mosquitoes" (Video, $7 \mathrm{~min}$.$) ,$ https://www.youtube.com/watch?v=_-5BvgP78aI\&t.

Bates, Marston. 1953. "Preface." In John Logan. The Sardinian Project: An Experiment in the Eradication of an Indigenous Malarious Vector. Baltimore: Johns Hopkins University Press.

BBC News. 2013. Can the buzz of mosquitoes be art? Nov. 1 at https://www.bbc.com/ news/av/entertainment-arts-24766729 on 21.10.2020.

Bhaumik, Soumyadeep. 2013. Aggressive Asian tiger mosquito invades Europe. Canadian Medical Association Journal, 185(10): E464-4.

Boomgard, Peter and Marjolein 't Hart. 2010. Globalization, environmental change, and social history: An introduction. International Review of Social History, 55(s18): 1-26.

Borrell, Brendan. 2009. Mosquitoes mate in perfect harmony. Nature. doi:10.1038/ news.2009.1167

Brand, Stewart. 1968. Whole Earth Catalog 1 Menlo Park, CA: The Portola Institute, 2.

Brown, Peter. 1998. Failure-as-success: Multiple meanings of eradication in the Rockefeller Foundation Sardinia project, 1946-1951. Parassitologia, 40(1-2): 117-130.

Carson, Rachel. 1962a. Speech to the Women's National Press Club (Dec. 5). Quoted in Shirley A. Briggs, "Rachel Carson: Her Vision and Her Legacy." In G. Marco, R. Hollingworth, W. Durham, eds. 1987. Silent Spring Revisited. Wash., D.C.: American Chemical Society, 7.

Carson, Rachel. 1962b. Silent Spring. Boston: Houghton Mifflin, 99.

Carter, Richard and Kamini Mendis. 2002. Evolutionary and historical aspects of the Burden of Malaria. Clinical Microbiology Reviews, 15(4): 564-594.

Conlon, Joe. 2011. Mosquito genocide. Nothing But Science at https://nothingbutscience .wordpress.com/tag/joe-conlon/ on 28.5.19.

Fang, Janet. 2010. A world without mosquitoes. Nature, 466:432-434.

Gallup, John Luke and Jeffrey Sachs. 2001. The economic burden of malaria. The American Journal of Tropical Medicine and Hygiene, 64(1,2): 85-96.

Hall, Marcus. 2010. Environmental imperialism in Sardinia: Pesticides and politics in the struggle against malaria. In Nature and History in Modern Italy, Marco Armiero and Marcus Hall, eds. Athens: Ohio University Press, 70-86.

Hall, Marcus, Philippe Forêt, Christoph Kueffer, Alison Pouliot and Caroline Wiedmer. 2015. Seeing the environment through the humanities: A new window on grand societal challenges. GAIA, 24(2): 134-136.

Jones, Richard. 2012. Mosquito. London: Reaction Books.

Kennedy, Donald and Marjorie Lucks. 1999. Rubber, blight, and mosquitoes: Biogeography meets the global economy. Environmental History, 4(3): 369-383.

Logan, John. 1953. The Sardinian Project: An Experiment in the Eradication of an Indigenous Malarious Vector. Baltimore: Johns Hopkins University Press. 
Méthot, Pierre-Olivier. 2012. Why do parasites harm their host? On the origin and legacy of Theobald Smith's 'law of declining virulence'. History and Philosophy of the Life Sciences, 34: 567.

Mosquito Reviews. 2020. Statistics for Mosquito-Borne Diseases and Deaths at https://mo squitoreviews.com/learn/disease-death-statistics/ on 21.10.2020.

Myers, Norman. 1993. Biodiversity and the precautionary principle. Ambio, 22(2/3): 74-79.

Packard, Randall M. 2007. The Making of a Tropical Disease: A Short History of Malaria. Baltimore: The Johns Hopkins University Press.

Patterson, Gordon. 2009. The Mosquito Crusades: A History of the American Anti-Mosquito Movement. New Brunswick: Rutgers University Press, 83.

Rose, Noah, et al. 2020. Climate and urbanization drive mosquito preference for humans. Current Biology, 30(18), https://doi.org/10.1016/j.cub.2020.06.092.

Roux, Olivier and Vincent Robert. 2019. Larval predation in malaria vectors and its potential implication in malaria transmission: An overlooked ecosystem service? Parasites \& Vectors, 12: 217.

Snounou, Georges and Nicolas White. 2004. The co-existence of plasmodium: Sidelights from falciparum and vivax malaria in Thailand. Trends in Parasitology, 20(7): 333-339.

Snow, Charles Percy. 1959. The Two Cultures and the Scientific Revolution. New York: Cambridge University Press.

Spielman, Andrew and Michael D'Antonio. 2001. Mosquito: The Story of Man's Deadliest Foe. New York: Hyperion.

Török, Edina, et al. 2020. Unmeasured side effects of mosquito control on biodiversity. European Journal of Ecology, 6.1: 71-76.

Winegard, Timothy C. 2019. Mosquito: A Human History of Our Deadliest Predator. New York: Hutton.

World Health Organization. 2014. Yellow fever global annual reported cases and YFV coverage, 1980-2014 at http://158.232.12.119/emergencies/yellow-fever/maps/arc hive/en/ on 21.10.2020. 